\title{
AMPLITUDE DE MOVIMENTO APÓS ARTROPLASTIA TOTAL DO JOELHO
}

\author{
RANGE OF MOTION AFTER TOTAL KNEE ARTHROPLASTY
}

\author{
lúcio Honório de Carvalho Júnior ${ }^{1}$, Cézar Augusto Costa de Castro², Matheus Braga Jacques Gonçalves ${ }^{3}$, \\ Leonardo Costa Martins Rodrigues ${ }^{4}$, Felipe Vieira Pinto da Cunha 4 , Fernando de Lima Lopes ${ }^{4}$
}

\section{RESUMO}

Objetivo: Avaliar a amplitude de movimentos (ADM) do joelho após seis meses de pós-operatório de artroplastia total, comparando-a com o seu valor pré-operatório. Material e Métodos: Foi analisada a amplitude de movimento de 80 artroplastias, com acompanhamento pós-operatório médio de 21,9 meses. Resultado: Não houve diferença significativa entre as médias pré e pós-operatória, de extensão $(p=0,09)$ e flexão $(p=0,47)$. Subdividindo os pacientes em dois grupos, naqueles com flexão pré-operatória menor ou igual a $90^{\circ}(n=17)$ observou-se aumento significativo da flexão $(p=0,000037)$. Naqueles com flexão pré-operatória maior ou igual a $120^{\circ}(n=31)$ ocorreu diminuição significativa da flexão $(p=0,000068)$. Quatro pacientes foram submetidos à manipulação, em média com 2,1 meses de pós-operatório. A média de flexão antes da manipulação e após seis meses dessa foi significativamente diferente $(p=0,0068)$. Já as médias de flexão pré-operatória e após seis meses de pós-operatório foram semeIhantes $(p=0,28)$. Conclusão: A flexão pré-operatória influencia de forma significativa a flexão após artroplastia total do joelho. Sua medida fornece ao cirurgião bom parâmetro para prevê-la.

Descritores: Amplitude de movimento; Artroplastia; Joelho.

\section{SUMMARY}

Objective: To evaluate knee range of motion (ROM) after at least six months postoperatively in total knee arthroplasty, by comparing it to baseline value. Materials and Methods: Ranges of motion of eighty arthroplasties were analyzed within an average follow-up of 21.9 months. Results: No significant differences between pre- and postoperative averages for extension $(p=0.09)$ and flexion $(p=0.47)$ were found. By dividing the patients into two groups, a significant flexion improvement was seen in those with pre-op flexion equal or smaller than $90^{\circ}(n=17)(p=0.000037)$. In the other group, a significant decrease in flexion was seen in those with pre-op flexion equal or greater than $120^{\circ}(n=31)(p=0.000068)$. Four patients have been submitted to knee manipulations within an average of 2.1 months postoperatively. The mean flexion rate before manipulation and after six months was significantly different $(p=0.0068)$. The mean pre-op flexion and after six months postoperatively was similar $(p=0.28)$. Conclusion: Preoperative flexion significantly influences the postop flexion in total knee arthroplasty. Its measurement provides the surgeon with a good parameter for previewing this.

Keywords: Range of motion; Arthroplasty; Knee.

\section{INTRODUÇÃO}

A artroplastia total do joelho (ATJ) tem como objetivo aliviar a dor, corrigir deformidades e permitir arco de movimento funcional, mantendo a estabilidade e a função do joelho para atividades cotidianas $^{(1)}$. A ATJ é procedimento eficaz para o tratamento da dor e para correção de deformidades associadas com a doença articular degenerativa ${ }^{(2,3)}$

Apesar das evoluções da técnica cirúrgica, dos implantes e do manejo pós-operatório, a rigidez continua a ser problema comum após ATJ(2). A amplitude de movimento (ADM) obtida no pós-operatório depende principalmente do seu valor no pré-operatório ${ }^{(3-6)}$. A idade e a morfologia do paciente, o diagnóstico e o modelo da prótese parecem não influenciá-la $a^{(3-5)}$.

O objetivo deste trabalho é avaliar a ADM do joelho de pacientes submetidos a ATJ comparando-as com a ADM pré-operatória.

\section{MATERIAL E MÉTODOS}

Entre junho de 1995 e julho de 2004 foram realizadas pelo autor principal, 137 ATJ em 127 pacientes no Hospital Madre Teresa (HMT) de Belo Horizonte. Através de revisão dos prontuários foram obtidos os valores da ADM pré-operatória e após seis meses da cirurgia. Cinqüenta e sete pacientes que não fizeram acompanhamento mínimo de seis meses ou que não tiveram os prontuários localizados, foram eliminados, totalizando 80 artroplastias avaliadas.

A média de idade dos pacientes foi 71,6 anos, variando entre 54 e 91 anos. Sessenta e nove $(86,25 \%)$ artroplastias foram realizadas em mulheres e 11 (13,75\%) em homens. Quarenta e seis (57,5\%) artroplastias foram realizadas do lado direito e 34 (42,5\%) do lado esquerdo. Todas as próteses foram cimentadas e com preservação do ligamento cruzado posterior. Foram utilizados quatro diferentes modelos: Duracon ${ }^{\circledR}$ - Howmedica ${ }^{\circledR}(46)$, Nexgen $^{\circledR}$ - Zimmer ${ }^{\circledR}(17)$, Search $^{\circledR}$ - Aesculap ${ }^{\circledR}(9)$ e Advantin ${ }^{\circledR}$ - Wright ${ }^{\circledR}(8)$. O diagnóstico foi osteoartrose em 76 (95\%) casos, artrite reumatóide em 3 (3,75\%) e osteonecrose em 1 (1,25\%). O acompanhamento pós-operatório médio foi 21,9 meses, sendo o maior 102 meses e o menor 6 meses.

Foi utilizado o teste $t$ de Student para comparação entre médias com valor de $p$ significativo se menor que 0,05.

O projeto de pesquisa foi aprovado pela comissão de ética em pesquisa do HMT.

\section{Trabalho realizado no Hospital Madre Teresa de Belo Horizonte.}

Endereço para correspondência: R. Olavo Carsalade Vilela, 264. Residencial Ipê da Serra, Nova Lima, MG, Brasil. CEP 34.000-100. E-mail: luciohcj@medicina.ufmg.br

1. Doutor em Ortopedia pela Universidade Federal de Sáo Paulo (UNIFESP), Professor Adjunto do Departamento do Aparelho Locomotor da Faculdade de Medicina da UFMG.

Médico Ortopedista do Hospital Madre Teresa (HMT) de Belo Horizonte.

2. Membro Titular da SBOT, especializando em Cirurgia do Joelho do HMT.

3. Membro titular da SBOT, especialista em Cirurgia do Joelho pelo HMT.

4. Médico residente de Ortopedia e Traumatologia do HMT. 


\section{RESULTADOS}

A extensão pré-operatória média foi $0,6^{\circ}$ com desvio padrão (DP) igual a 2,53 e a pós-operatória $0,1^{\circ}(\mathrm{DP}=0,78)$. Não se observou diferença significativa $(p=0,09)$ entre as médias de extensão pré e pós-operatórias. A flexão pré-operatória média foi $107,1^{\circ}$ $(\mathrm{DP}=15,95)$ e a pós-operatória $108,6^{\circ}(\mathrm{DP}=11,87)$, não se observando diferença significativa entre elas $(p=0,47)$.

Analisando os resultados sob outro ponto de vista, os pacientes foram subdivididos em três grupos: Grupo 1 - aqueles com flexão pré-operatória menor ou igual a $90^{\circ}$, Grupo 2 - aqueles com flexão pré-operatória maior ou igual a $120^{\circ}$ e Grupo 3 - aqueles submetidos à manipulação. Os diferentes modelos de prótese foram distribuídos uniformemente entre os grupos, não sendo considerado fator de confusão na análise dos resultados.

Entre os pacientes com flexão pré-operatória menor ou igual a $90^{\circ}$ $(\mathrm{n}=17)$, a flexão pré-operatória média foi $85^{\circ}(\mathrm{DP}=8,66)$ e a pósoperatória $100,5^{\circ}(\mathrm{DP}=9,88)$. Observou-se diferença significativa entre essas médias ( $p=0,000037$ ).

Para os pacientes com flexão pré-operatória maior ou igual a $120^{\circ}$ $(n=31)$ a média de flexão pré-operatória foi $123,5^{\circ}(D P=4,88)$ e a pós-operatória $112,8^{\circ}(\mathrm{DP}=13,10)$. Essa diferença mostrou-se significativa $(p=0,000068)$.

Seis artroplastias (7,5\%) evoluíram com rigidez pós-operatória (Grupo 3). Em dois pacientes aceitou-se ADM de $80^{\circ} \mathrm{e}$, nos demais $(n=4)$ foi realizada manipulação, em média com 2,1 meses de pósoperatório. A média de flexão desses pacientes foi $107,5^{\circ}(\mathrm{DP}=15)$ no pré-operatório, $54,5^{\circ}(\mathrm{DP}=16,76)$ antes da manipulação e $94,7^{\circ}(\mathrm{DP}=16,76)$ após seis meses de pós-operatório. A análise estatística mostrou diferenças significativas entre as médias de flexão pré-operatória e antes da manipulação $(p=0,000046)$ e entre as médias de flexão antes da manipulação a após seis meses de pós-operatório $(p=0,0068)$. Não se observou diferença significante entre a flexão pré-operatória e após seis meses de pós-operatório $(p=0,28)$.

Os resultados encontram-se sumarizados na Tabela 1

\section{DISCUSSÃO}

\begin{tabular}{|c|c|c|c|c|}
\hline & $\begin{array}{c}\text { Pré - } \\
\text { operatório }\end{array}$ & $\begin{array}{c}\text { Pré - } \\
\text { manipulaçăo }\end{array}$ & $\begin{array}{c}\text { Após } 6 \\
\text { meses P.O. }\end{array}$ & $\begin{array}{c}\text { Valor } \\
p\end{array}$ \\
\hline Extensão $(n=80)$ & $\begin{array}{c}0,6^{\circ} \\
\mathrm{DP}=2,53\end{array}$ & $-\infty$ & $\begin{array}{c}0,1^{\circ} \\
\mathrm{DP}=0,78\end{array}$ & 0,093 \\
\hline Flexão $(n=80)$ & $\begin{array}{c}107,1^{\circ} \\
D P=15,95\end{array}$ & $\ldots$ & $\begin{array}{c}108,6^{\circ} \\
D P=11,87\end{array}$ & 0,475 \\
\hline $\begin{array}{l}\text { Flexão - grupo } 1(\mathrm{n}=17) \\
\text { Obs.: flexão pré-operatória } 90^{\circ}\end{array}$ & $\begin{array}{c}85^{\circ} \\
\mathrm{DP}=8,66\end{array}$ & $\ldots$ & $\begin{array}{c}100,5^{\circ} \\
D P=9,88\end{array}$ & 0,000037 \\
\hline $\begin{array}{l}\text { Flexão-grupo } 2(n=31) \\
\text { Obs.: flexão pré-operatória } 120^{\circ}\end{array}$ & $\begin{array}{c}123,5^{\circ} \\
D P=4,88\end{array}$ & - & $\begin{array}{c}112,8^{\circ} \\
D P=13,10\end{array}$ & 0,000068 \\
\hline $\begin{array}{l}\text { Flexão - grupo } 3(n=4) \\
\text { Obs.: pacientes submetidos à manipulação }\end{array}$ & $\begin{array}{l}107,5^{\circ} \\
D P=15\end{array}$ & $\begin{array}{c}54,5^{\circ} \\
D P=16,76\end{array}$ & $-\overline{-O}^{-}$ & 0,000046 \\
\hline $\begin{array}{l}\text { Flexão - grupo } 3(n=4) \\
\text { Obs.: pacientes submetidos à manipulação }\end{array}$ & $\cdots$ & $\begin{array}{c}54,5^{\circ} \\
D P=16,76\end{array}$ & $\begin{array}{c}94,7^{\circ} \\
D P=16,76\end{array}$ & 0,006862 \\
\hline $\begin{array}{l}\text { Flexão - grupo } 3(n=4) \\
\text { Obs.: pacientes submetidos à manipulação }\end{array}$ & $\begin{array}{l}107,5^{\circ} \\
D P=15\end{array}$ & - & $\begin{array}{c}94,7^{\circ} \\
D P=16,76\end{array}$ & 0,281 \\
\hline
\end{tabular}

Tabela 1 - Comparação entre as diversas amplitudes de movimento, com média, Desvio Padrão (DP) e valor $p$.
AADM do joelho necessária para atividades diárias foi determinada por estudos biomecânicos e de análise de marcha. Laubenthal et al.. ${ }^{(7)}$ mostraram que os pacientes requerem $67^{\circ}$ de flexão durante a fase de balanço da marcha, $83^{\circ}$ para subir degraus, $100^{\circ}$ para descê-los, $93^{\circ}$ para levantar de cadeira e entre $71^{\circ}$ e $117^{\circ}$ para pegar um objeto no chão.

Concordando com os achados de Schurman e Rojer ${ }^{(6)}$ que compararam cinco tipos diferentes de prótese de joelho em relação à sua ADM pós-operatória, esse estudo não encontrou diferença significativa entre os quatro tipos utilizados.

Esse estudo confirma que a flexão pré-operatória é parâmetro útil para prognosticar a flexão pós-operatória após ATJ, concordando com os achados de Ritter e Stringer ${ }^{(3)}$, Parsley et al. ${ }^{(5)}$, Harvey et al. ${ }^{(4)}$ e Schurman e Rojer ${ }^{(6)}$. A média de flexão pré-operatória foi semelhante à média após seis meses da cirurgia $(p=0,47)$. Ritter e Stringer ${ }^{(3)}$ e Parsley et al. ${ }^{(5)}$ afirmaram que pacientes com pouca flexão antes da cirurgia tendem a ganhar movimento e os que têm flexão maior que a média tendem a perdê-la. Essa tendência foi confirmada por esse estudo. Os pacientes com flexão menor ou igual a $90^{\circ}$ graus ganharam movimento $(p=0,000037)$ e os com flexão maior ou igual a $120^{\circ}$ o perderam ( $\left.p=0,000068\right)$.

Apesar dos avanços técnicos, a rigidez continua a ser complicação freqüente após ATJ. Não existe critério universalmente aceito para seu diagnóstico. Rigidez é definida como amplitude de movimento inadequado para as atividades diárias, resultando em limitações funcionais ${ }^{(2)}$. A incidência varia na literatura entre $8 \%$ e $12 \%{ }^{(8)}$. Entre os pacientes desse trabalho, seis $(7,5 \%)$ evoluíram com flexão menor que $90^{\circ}$ no pós-operatório.

Fox e Ross ${ }^{(9)}$ e Esler et al. ${ }^{(10)}$ demonstraram que os pacientes ganham movimento após a manipulação alcançando flexão semelhante à préoperatória, evoluindo da mesma forma que os demais pacientes. Essas observações são confirmadas por esse estudo. Entre os pacientes submetidos a manipulação do joelho, as médias de flexão antes da manipulação e após seis meses de pósoperatório foram significativamente diferentes ( $p=0,0068)$. Já as médias de flexão pré-operatória e após seis meses de pósoperatório foram semelhantes $(p=0,28)$.

\section{CONCLUSÃO}

Na análise da amplitude de movimento após ATJ, a flexão pré-operatória influenciou de forma significativa a flexão pós-operatória. Sua medida fornece ao cirurgião bom parâmetro para predizer a flexão após a artroplastia.

\section{REFERÊNCIAS BIBLIOGRÁFICAS}

1. Buechel FF, Pappas MJ. Long-term survivorship analysis of cruciate-sparing versus cruciatesacrificing knee prostheses using meniscal bearings. Clin Orthop 1990: 260:162-9.

Bong MR, Cesare PE. Rigidez após artroplastia total de joelho. J Am Acad Orthop Surg, 2004 2: 227-34. (Edição Brasileira)

3. Ritter MA, Stringer EA. Predictive range of motion after total knee replacement. Clin Orthop 1979; 143: 115-9.

4. Harvey IA, Barry K, Kirby SP Johnson R, Elloy MA. Factors affecting the range of movement Harvey IA, Barry K, Kirby SP Johnson R, Elloy MA. Factors affecting
of total knee arthroplasty. J Bone Joint Surg Br 1993; 75: 950-5.

5. Parsley BS, Engh GA, Dwyer KA. Preoperative flexion. Does it influence postoperative flexion after posterior-cruciate-retaining total knee arthroplasty? Clin Orthop 1992; 275:204-10.
6. Schurman DJ, Rojer DE. Total knee arthroplasty. Range of motion across five systems. Clin Orthop 2005; 430: 132-7.

Laubenthal KN, Smidt GL, Kettelkamp DB. A quantitative analysis of knee motion during activities of daily living. Phys Ther 1972; 52:34-43. 8. Scranton PE Jr. Management of knee pain and stiffness after total knee arthroplasty. J Arthro-

9. Fox JL, Ross R. The role of manipulation following total knee replacement. J Bone Joint Surg Am 1981; 63:357.

10. Esler CN, Lock K, Harper WM, Gregg PJ. Manipulation of total knee replacements. Is the flexion gained retained? J Bone Joint Surg Br 1999; 81:27-9. 